\title{
Hurry up and slow down: Lessons in drug development from the COX-2 inhibitors
}

\author{
Mark Stafford-Smith MD CM FRCPC
}

A Google ${ }^{\mathrm{TM}}$ search performed for this editorial using the terms "COX-2 inhibitor" and "lawyer" had 292,000 hits. Certainly a swarm of legal attention has marked the end of a six-year period that saw the introduction of cyclooxygenase (COX)-2 inhibitor analgesics (1999), their growth to include millions of prescriptions annually worldwide at an estimated cost exceeding \$Cdn 6 billion, and the equally sudden almost complete disappearance of these agents from the market due to safety concerns. The apparent blind spot in drug development methods exposed by this course of events has raised serious concerns. How did a process designed to provide assurance of safety and efficacy in the search for new drugs simultaneously fail in so many countries, including Canada, the United States, and European countries? The consequences of this misadventure have resulted in patient harm, physician alarm and concern, and litigious actions that are still playing out for the large drug companies. ${ }^{1,2}$ Furthermore, these events have eroded confidence regarding already available drugs (e.g., aprotinin), ${ }^{3}$ questioning the sufficiency of safety evaluations based on the large randomized double-blind controlled trials traditionally used as evidence for regulatory approval. There are obvious and important implications for all disciplines of medicine, including anesthesia and perioperative medicine.

Undeniably, in recent years there has been increasing pressure to speed up drug development, both from the medical field and the drug industry. Physicians see needless suffering occurring while better therapies wade through perceived excessive red tape, while drug companies also protest that unnecessary delays in approval add cost and shorten the patent-protected marketing period. In the market of pain relief, COX-2 inhibitors were envisioned to be more ideal compared to available analgesics. Non-steroidal anti-inflammato- ry drugs (NSAIDs) provide good pain relief but cause an estimated 15,000 deaths and over 100,000 hospitalizations each year in the United States alone, primarily from gastrointestinal complications. Similarly, respiratory depression and abuse complicate the use of opioid analgesic agents and cause significant morbidity and mortality.

Thus COX-2 inhibitors emerged, appearing to safely tease apart therapeutic benefit from side effect by more selectively targeting the analgesic properties of NSAIDs. Basic science research was important in this part of the drug discovery process. Animal studies identified two subtypes of COX ${ }^{4}$ the enzyme that mediates the generation of prostacyclin and thromboxane from arachadonic acid, that is inhibited nonselectively by NSAIDs. Moreover, selective COX-2 subtype inhibitors appeared to offer NSAID-like analgesia without many of the typical NSAID side effects mediated by the COX-1 subtype, including the most serious gastrointestinal complications. Even more promising was the identification of COX-2 subtype selective substances that were non-toxic to humans.

After an uneventful course through early human studies, large randomized double-blind COX-2 trials were initiated. Standard safety recording occurred during these trials, and subtle evidence of increased cardiovascular complications was noticed, but not looked upon as a major issue. Indeed, a Bayesian approach to the search for significant findings demands more than a simple $P<0.05$, and background evidence did not yet justify concern - after all, aspirin is cardioprotective and the less-selective NSAIDs (e.g., naproxen) do not increase cardiovascular risk; since COX-2 inhibitor side effects were expected to be a subset of NSAID complications, little heed was taken of this "incidental finding". Again, the role of basic science was important; animal studies were already identifying unanticipated prothrombotic effects from COX-2

From the Department of Anesthesiology, Duke University Medical Center, Durham, North Carolina, USA.

Address correspondence to: Dr. Mark Stafford-Smith, Associate Professor of Anesthesiology, Director, Fellowship Education, Director, Cardiothoracic Anesthesia \& Critical Care Medicine Fellowship, Department of Anesthesiology, Duke University Medical Center, Box 3094 DUMC, Durham, NC 27710, USA. Phone: 919-681-5046; Fax: 919-681-8993; E-mail: staff002@mc.duke.edu 
inhibition due to reduced prostacyclin generation. ${ }^{5}$ Furthermore, COX-2 inhibitors lack the antiplatelet effects of aspirin, and even favour vasoconstrictive effects. Nonetheless, with analgesic efficacy confirmed through the Celecoxib Long-Term Arthritis Safety Study (CLASS) ${ }^{6}$ and Vioxx Gastrointestinal Outcomes Research (VIGOR) study, ${ }^{7}$ the first COX-2 inhibitor drugs - refoecoxib and celecoxib were approved in the United States in 1999, and soon thereafter in Canada and Europe. ${ }^{1}$

Other post-approval randomized controlled trials were (fortunately) initiated that involved routine safety assessments - notably, these were not mandated to further assess cardiovascular risk, but were industryinitiated to assess COX-2s for other potential indications. Cyclooxygenase-2s were evaluated as alternate therapies in the Adenoma Prevention with Celecoxib (APC) Trial $^{8}$ and Alzheimer Disease Anti-inflammatory Prevention (ADAPT) Trial. ${ }^{9}$ It was the increased cardiovascular complications in interim reviews of safety data for these two trials, supported by evidence from previous studies, that caused their Data Safety Monitoring Boards to advise a full safety review of cardiovascular events. These reviews characterized the increased cardiovascular risk from the whole class of drugs that had previously been missed..$^{8,10,11}$

As the smoke clears, the current availability of COX-2 inhibitors differs by country, involving variable suspension of sales and prescribing restrictions. ${ }^{12}$ Of the class of selective COX-2 inhibitor drugs, celecoxib is the agent with the least evidence of harm and is still available with restrictions in some countries. ${ }^{13}$ The difference between celecoxib and the other agents may be due to its relatively modest COX-2 vs COX-1 subtype selectivity compared to the other agents (30:1 vs > 300:1). Nonetheless, celecoxib has also been incriminated, and harmful effects may simply be less striking.

The major points that can be drawn from this episode in drug development are twofold. The first is an acceptance that the drug approval process should "slow down" - despite reasonable decisions, postapproval surveillance of drug safety was flawed in this case - how long would it have taken to demonstrate these complications, had post-marketing studies for other indications not come to the rescue? Revisions of the regulatory safety assessment process will need to be adopted that improve the chances of identifying changes in the incidence of common complex conditions (e.g., hypertension, diabetes, stroke) in addition to the likelihood of rare catastrophic events (e.g., aplastic anemia). Any modifications to the process that emerge will have to maintain a delicate balance between improvements and not disturbing existing strengths, while bringing new agents to market as quickly as possible. In order to avoid unneeded delays, possible solutions may require probationary periods to accumulate larger safety cohorts before unrestricted use of an agent is endorsed. Certainly, COX-2 inhibitors are not the first or the longest available agents to highlight this blind spot in drug surveillance - perimenopausal estrogen therapy has been available for decades but was only recently recognized to have unexpectedly high cardiovascular risk. ${ }^{14} \mathrm{~A}$ second major point is to recognize the importance of basic science investigation in drug development. More attention to laboratory findings may have prompted careful inspection of the safety data from human COX-2 inhibitor trials during their approval process. It is still possible that with better understanding of these agents, a safe role for their potent analgesic effects may ultimately be found. It would be unfortunate to lose such well demonstrated analgesic benefits as part of a multi-modal analgesic strategy for the post-operative period.

In conclusion, the numerous national institutions charged with drug approval in their respective countries have been highly successful in identifying effective drugs to treat medical problems, largely through the use of large randomized clinical trials. However, the COX-2 saga has served to demonstrate that this approach, based on studies powered to demonstrate effectiveness, no longer meets societal expectations for a comprehensive review of acceptable drug safety. The lessons that emerged from the blindspots uncovered during the COX-2 crisis will be useful to guide modifications to the drug approval process that will further strengthen existing approaches to safety review. While therapies will always be vulnerable to side effects, evolution of this process should further advance the overall goal of providing optimal care and serving the best interests of our patients. 


\section{Se hâter et ralentir : les leçons à tirer de la pro- duction des inhibiteurs de la COX-2}

La recherche préparatoire au présent éditorial, réalisée dans Google ${ }^{\mathrm{TM}}$ selon les termes «COX-2 inhibitor» et «lawyer», a affiché 292000 cibles. Il est certain que beaucoup de questions juridiques ont marqué la fin des six ans qui ont vu l'apparition des analgésiques inhibiteurs de la cyclo-oxygénase (COX)-2 (1999), leur croissance qui a atteint des millions de prescriptions annuelles dans le monde pour un coût estimé à plus de six milliards de dollars canadiens et leur disparition aussi soudaine, et presque complète, à cause des inquiétudes reliées à leur innocuité. La faiblesse apparente des méthodes de développement des médicaments, exposée par cette suite d'événements, a soulevé de sérieuses inquiétudes. Comment un processus conçu pour fournir sécurité et efficacité dans la recherche de nouveaux médicaments a-t-il simultanément échoué dans tant de pays, dont le Canada, les États-Unis et des pays européens ? Finalement, cette mésaventure a nui aux patients, alarmé et préoccupé les médecins et provoqué des litiges non réglés encore pour les grandes compagnies pharmaceutiques. ${ }^{1,2}$ Ces événements ont aussi miné la confiance face aux médicaments déjà sur le marché, comme l'aprotinine, ${ }^{3}$ et remis en question la qualité de la sécurité des évaluations habituellement fondées sur de grands essais randomisés et contrôlés en double insu pour une approbation réglementaire. Les implications sont évidentes et importantes pour toutes les disciplines médicales, dont l'anesthésie et la médecine périopératoire.

On ne peut nier qu'au cours des dernières années, une pression croissante du monde médical autant que de l'industrie pharmaceutique s'est exercée pour hâter le développement de médicaments. Les médecins sont témoins de souffrances inutiles alors que de meilleurs traitements sont retardés par une bureaucratie jugée excessive et que les compagnies pharmaceutiques affirment que les délais indus menant à l'approbation ajoutent au coût et raccourcissent la période de commercialisation protégée par le brevet. Parmi les analgésiques, les inhibiteurs de la COX-2 ont été perçus comme supérieurs aux médicaments existants. Les antiinflammatoires non stéroïdiens (AINS) sont efficaces, mais causent environ 15000 décès et plus de 100000 hospitalisations chaque année aux États-Unis, surtout pour des complications gastro-intestinales. Aussi, la dépression respiratoire et l'abus compliquent l'usage des analgésiques opioïdes et causent une morbidité et une mortalité significatives.

Alors arrivent les inhibiteurs de la COX-2 qui semblent dissocier sans danger les effets thérapeutiques des effets secondaires en ciblant plus sélectivement les propriétés analgésiques des AINS. La recherche en science fondamentale a été importante dans cette partie de la découverte des médicaments. Des études sur les animaux ont révélé deux sous-types de $\mathrm{COX},{ }^{4}$ l'enzyme par lequel sont générées la prostacycline et la thromboxane à partir de l'acide arachidonique qui est inhibé non sélectivement par les AINS. Qui plus est, les inhibiteurs d'un sous-type sélectif de COX2 paraissaient offrir une analgésie comme celle des AINS sans nombre de leurs effets secondaires typiques originant du sous-type de COX-1, dont les plus graves complications gastro-intestinales. Plus prometteuse encore a été la détection de substances sélectives du sous-type COX-2 qui n'étaient pas toxiques pour les humains.

Les premières études des COX-2 sur les humains étant sans incident, de grands essais randomisés à double insu ont commencé. Des mesures standards de la sécurité et de minces preuves de complications cardiovasculaires accrues ont été notées, mais non vues comme majeures. Il faut dire qu'une approche Bayesienne de la recherche de résultats significatifs demande plus qu'un simple $P<0,05$, et la preuve contextuelle ne justifiait pas d'inquiétudes - après tout, l'aspirine est cardioprotectrice et les AINS les moins sélectifs, comme le naproxène, n'augmentent pas le risque cardiovasculaire ; or, on pensait que les effets secondaires des inhibiteurs de la COX-2 étaient un sous-groupe de complications des AINS, donc on a fait peu de cas de ce «résultat accessoire». Encore une fois, le rôle de la science fondamentale a été important ; les études sur les animaux montraient déjà des effets prothrombotiques inattendus de l'inhibition de la COX-2 à cause de la génération réduite de prostacycline..$^{5}$ De plus, les inhibiteurs de la COX-2 n'ont pas les effets antiplaquettaires de l'aspirine et favorisent même les effets vasoconstricteurs. Mais, avec la confirmation de l'efficacité analgésique par la Celecoxib Long-Term Arthritis Safety Study (CLASS) ${ }^{6}$ et la Vioxx Gastrointestinal Outcomes Research (VIGOR), ${ }^{7}$ les premiers inhibiteurs de la COX-2, le rofécoxib et le célécoxib, ont été acceptés aux États-Unis en 1999 et peu après au Canada et en Europe. ${ }^{1}$

Heureusement, d'autres essais randomisés ont suivi l'acceptation des médicaments; ils comportaient des 
évaluations de routine de la sécurité et, fait à noter, n'avaient pas été demandés pour évaluer plus avant le risque cardiovasculaire, mais lancés par l'industrie pour évaluer d'autres indications possibles des COX-2. Les cyclo-oxygénase- 2 ont été évaluées comme traitement de remplacement dans le Adenoma Prevention with Celecoxib (APC) Trials et l'Alzheimer Disease Anti-inflammatory Prevention (ADAPT) Trial. ${ }^{9} \mathrm{Ce}$ sont les complications cardiovasculaires croissantes découvertes lors des examens provisoires des données sur la sécurité de ces deux essais, corroborées par des preuves d'études antérieures, qui ont amené les comités de surveillance de la sauvegarde des données à conseiller une revue des incidents cardiovasculaires en regard de la sécurité. Cette revue a défini le risque cardiovasculaire croissant venant de cette catégorie de médicaments, risque non perçu antérieurement. ${ }^{8,10,11}$

Maintenant que la situation se stabilise, on voit que la disponibilité des inhibiteurs de la COX-2 diffère d'un pays à l'autre, allant de suspensions variables des ventes aux prescriptions restreintes. ${ }^{12}$ Des médicaments inhibiteurs sélectifs de la COX-2, le célécoxib présente le moins de preuve d'effet nuisible et est toujours offert avec des restrictions dans certains pays. ${ }^{13}$ La différence entre le célécoxib et les autres peut dépendre de sa sélectivité relativement modeste des sous-types COX-2 vs COX-1, en comparaison des autres agents (30:1vs > 300:1). Cependant, le célécoxib a aussi été mis en cause, mais ses effets nocifs sont peut-être tout simplement moins frappants.

Deux points majeurs sont à retenir du développement de ces médicaments. Le premier est d'accepter que le processus d'approbation d'un médicament doive «être ralenti» - malgré des décisions honnêtes, la pharmacovigilance suivant son approbation a été défectueuse dans ce cas - combien de temps aurait-il fallu pour démontrer ces complications si les études post-commercialisation n'étaient venues à la rescousse? Des révisions du processus d'évaluation réglementaire de la sécurité devront être adoptées pour améliorer les chances de reconnaître les changements d'incidence d'affections courantes complexes, comme l'hypertension, le diabète, l'accident vasculaire, en plus de la possibilité d'événements catastrophiques rares comme l'anémie aplastique. Toute modification du processus devra maintenir un équilibre délicat entre les améliorations et l'absence de perturbation des mécanismes jugés efficaces, tout en produisant de nouveaux médicaments le plus rapidement possible. Pour éviter des délais inutiles, on pourrait exiger des périodes d'essais qui réuniraient de plus grandes cohortes sur l'innocuité avant que l'usage libre d'un médicament soit approuvé. Les inhibiteurs de la
COX-2 ne sont certainement pas les premiers ou les plus anciens médicaments à montrer une faille dans la pharmacovigilance - l'œstrogénothérapie périménopausique a été offerte pendant des décennies, mais n'a révélé que récemment des risques cardiovasculaires élevés. ${ }^{14}$ Un second point majeur est de reconnaître l'importance de la recherche en science fondamentale dans le développement des médicaments. Une plus grande attention aux résultats de laboratoire aurait pu hâter l'inspection minutieuse des données sur la sécurité à partir des essais sur les inhibiteurs de la COX-2 humaine pendant leur processus d'approbation. Il est encore possible, par une connaissance plus approfondie de ces médicaments, de leur découvrir finalement des effets analgésiques inoffensifs puissants. Il serait malheureux de perdre des avantages analgésiques si bien démontrés dans le cadre d'une stratégie analgésique postopératoire multimodale.

En conclusion, les nombreuses institutions nationales chargées d'approuver les médicaments dans leur pays respectif ont bien réussi à identifier les médicaments efficaces pour traiter les problèmes médicaux, en grande partie grâce aux grands essais cliniques randomisés. Cependant, la saga de la COX-2 a servi à démontrer que cette approche, fondée sur des études de recherche d'efficacité, ne répond plus aux attentes sociétales d'une revue complète de la sécurité acceptable des médicaments. Les leçons tirées des lacunes découvertes lors de la crise de la COX-2 pourront guider les modifications à apporter à l'approbation des médicaments, ce qui renforcera davantage les approches existantes à la revue de l'innocuité. Même si les traitements auront toujours des effets secondaires, l'évolution de ce processus devrait faire progresser l'objectif général de fournir les meilleurs soins et de servir les meilleurs intérêts de nos patients.

\section{References}

1 Drazen JM. COX-2 inhibitors--a lesson in unexpected problems (Editorial). N Engl J Med 2005; 352: 11312.

2 Psaty BM, Furberg CD. COX-2 inhibitors--lessons in drug safety (Editorial). N Engl J Med 2005; 352: 1133-5.

3 Mangano DT, Tudor IC, Dietzel C; Multicenter Study of Perioperative Ischemia Research Group: Ischemia Research and Education Foundation. The risk associated with aprotinin in cardiac surgery. N Engl J Med 2006; 354: 353-65.

4 Tanaka $\Upsilon$, Ward SL, Smith WL. Immunochemical and kinetic evidence for two different prostaglandin $\mathrm{H}$ prostaglandin $\mathrm{E}$ isomerases in sheep vesicular gland microsomes. J Biol Chem 1987; 262: 1374-81. 
5 McAdam BF, Catella-Lawson F, Mardini IA, Kapoor $S$, Lawson JA, FitzGerald GA. Systemic biosynthesis of prostacyclin by cyclooxygenase (COX)-2: the human pharmacology of a selective inhibitor of COX-2. Proc Natl Acad Sci USA 1999; 96: 272-7.

6 Mukherjee D, Nissen SE, Topol EJ. Risk of cardiovascular events associated with selective COX-2 inhibitors. JAMA 2001; 286: 954-9.

7 Bombardier C, Laine L, Reicin A, et al. Comparison of upper gastrointestinal toxicity of rofecoxib and naproxen in patients with rheumatoid arthritis. VIGOR Study Group. N Engl J Med 2000; 343: 1520-8.

8 Solomon SD, McMurray JJ, Pfeffer MA, et al.; Adenoma Prevention with Celecoxib (APC) Study Investigators. Cardiovascular risk associated with celecoxib in a clinical trial for colorectal adenoma prevention. N Engl J Med 2005; 352: 1071-80.

9 Martin BK, Meinert CL, Breitner JC; ADAPT Research Group. Double placebo design in a prevention trial for Alzheimer's disease. Control Clin Trials 2002; 23: 93-9.

10 Bresalier RS, Sandler RS, Quan H, et al.; Adenomatous Polyp Prevention on Vioxx (APPROVe) Trial

Investigators. Cardiovascular events associated with rofecoxib in a colorectal adenoma chemoprevention trial. N Engl J Med 2005; 352: 1092-102.

11 Nussmeier NA, Whelton AA, Brown MT, et al. Complications of the COX-2 inhibitors parecoxib and valdecoxib after cardiac surgery. N Engl J Med 2005; 352: 1081-91.

12 Konstantinopoulos PA, Lehmann DF. The cardiovascular toxicity of selective and nonselective cyclooxygenase inhibitors: comparisons, contrasts, and aspirin confounding. J Clin Pharmacol 2005; 45: 742-50.

13 Brophy JM. Cardiovascular risk associated with celecoxib (Letter, reply). N Engl J Med 2005; 352: 264850.

14 Rossouw JE, Anderson GL, Prentice RL, et al.; Writing Group for the Women's Health Initiative Investigators. Risks and benefits of estrogen plus progestin in healthy postmenopausal women: principal results from the Women's Health Initiative randomized controlled trial. JAMA 2002; 288: 321-33. 